\title{
Rhinolithiasis: An Unusual Misdiagnosed Entity
}

\author{
Puneeth S Nayak ${ }^{1}$, Samanvaya Soni ${ }^{2}$, Manasi B Manure ${ }^{3}$, Tejaswini J Suresh ${ }^{4}$
}

\begin{abstract}
Aim: To assess the usefulness of endoscopy in the initial visit for nasal obstruction in the outpatient department.

Background: Rhinolith as the name suggests is stone formation in the nose. They are formed by gradual deposition of calcium and magnesium salts in a manner to encrust an endogenous (thick mucus, blood clot, and ectopic teeth) or exogenous (seed and cotton fiber) nidus of origin. The first documented case of the pathology was reported by Barthdinin in 1654 . Since it is a slow-growing entity, it remains asymptomatic for a long period and often comes to light with presentation of unilateral nasal obstruction, foul smelling nasal discharge, cacosmia, and epistaxis. With its ability to cause rhinosinusitis and bony erosions of septum, lateral nasal wall, and hard palate, they are typically misdiagnosed.

Case description: A young patient came with the history of nasal obstruction on the left side for the past 1 year. There was an intermittent history of a yellow, thick, foul-smelling, blood-stained nasal discharge. Nasal endoscopic examination revealed an irregular, dark, stony mass covered with secretions confirming a rhinolith, which was removed piecemeal.

Conclusion: Considered as the cause of nasal obstruction and cacosmia, and many a times present in patients who have nasal complaints, delay in the diagnosis and treatment should be avoided. Rigid nasal endoscopy is the most important method to be used in diagnosis and treatment of rhinolith.

Clinical significance: Presentation of rhinolith and diagnostic dilemma are discussed in this article so as to enable the attending clinician to be aware of this still prevailing condition and to prevent complications, such as oroantral and oronasal fistulas, septal perforation, palatal perforation, bony destruction of the maxillary sinus, frontal osteomyelitis, and epidural abscess.
\end{abstract}

Keywords: Epistaxis, Foul smelling discharge, Rhinolith, Unilateral nasal obstruction.

Otorhinolaryngology Clinics: An International Journal (2019): 10.5005/jp-journals-10003-1329

\section{BACKGROUND}

Rhinolith as the name suggests is stone formation in the nose. They are formed by gradual deposition of calcium and magnesium salts in a manner to encrust an endogenous (thick mucus, blood clot, and ectopic teeth) or exogenous (seed and cotton fiber) nidus of origin. The first documented case of the pathology was reported by Barthdinin in 1654 . ' Since it is a slow-growing, entity it remains asymptomatic for a long period and often comes to light with presentation of unilateral nasal obstruction, foul-smelling nasal discharge, cacosmia, and epistaxis. With its ability to cause rhinosinusitis and bony erosions of septum, lateral nasal wall, and hard palate, they are typically misdiagnosed. ${ }^{2}$

\section{Case Description}

An otherwise healthy 22-year-old male patient presented with the complaint of nasal obstruction on the left side for the past 1 year. There was an intermittent history of a yellow, thick, foul-smelling, blood-stained nasal discharge. There was no history suggestive of any foreign body in the nose. The patient had old hospital records of being diagnosed with allergic rhinitis and rhinosinusitis, and despite his compliance with the treatment the symptoms failed to resolve. On anterior rhinoscopic examination, only thick mucopurulent discharge was seen in left nasal cavity. On computed tomography (CT) scan of paranasal sinuses, a $15 \times 11 \mathrm{~mm}$ calcified hyper dense mass was situated in the left nasal cavity along the floor abutting the septum and inferior turbinate corresponding to the anterior end of middle turbinate as shown in Figure 1. The patient was subjected to nasal endoscopic examination under local anesthesia. After suctioning the discharge, an irregular, dark, stony mass covered with secretions was present in the left nasal cavity, which

\footnotetext{
${ }^{1-4}$ Department of ENT, Jawaharlal Nehru Medical College, Belagavi, Karnataka, India
}

Corresponding Author: Samanvaya Soni, Department of ENT, Jawaharlal Nehru Medical College, Belagavi, Karnataka, India, Phone: +91 8722036108, e-mail: sonisamanvaya@gmail.com

How to cite this article: Nayak PS, Soni S, Manure MB, et al. Rhinolithiasis: An Unusual Misdiagnosed Entity. Int J Otorhinolaryngol Clin 2019;11(1):24-26.

Source of support: Nil

Conflict of interest: None

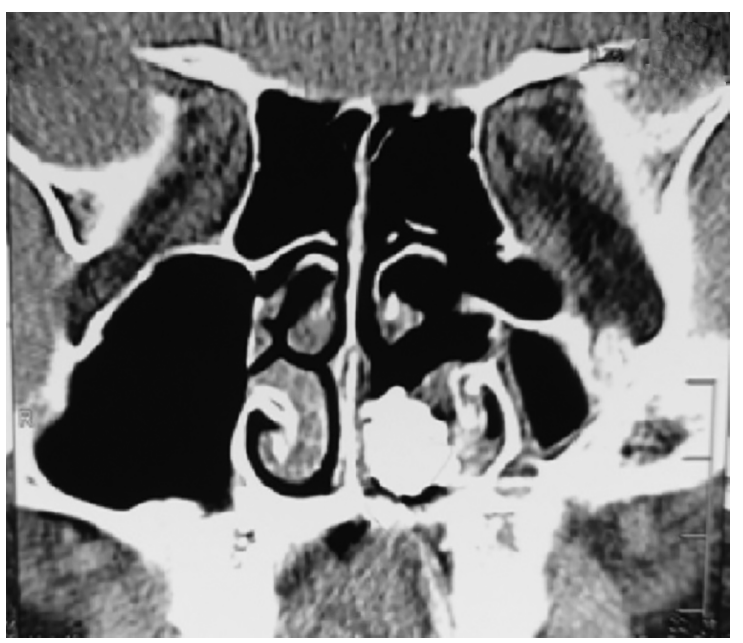

Fig. 1: CT-PNS coronal cut at the level of anterior end of middle turbinate. Presence of hyperdense homogeneous irregular mass in between the inferior turbinate and septum

() The Author(s). 2019 Open Access This article is distributed under the terms of the Creative Commons Attribution 4.0 International License (https://creativecommons. org/licenses/by-nc/4.0/), which permits unrestricted use, distribution, and non-commercial reproduction in any medium, provided you give appropriate credit to the original author(s) and the source, provide a link to the Creative Commons license, and indicate if changes were made. The Creative Commons Public Domain Dedication waiver (http://creativecommons.org/publicdomain/zero/1.0/) applies to the data made available in this article, unless otherwise stated. 


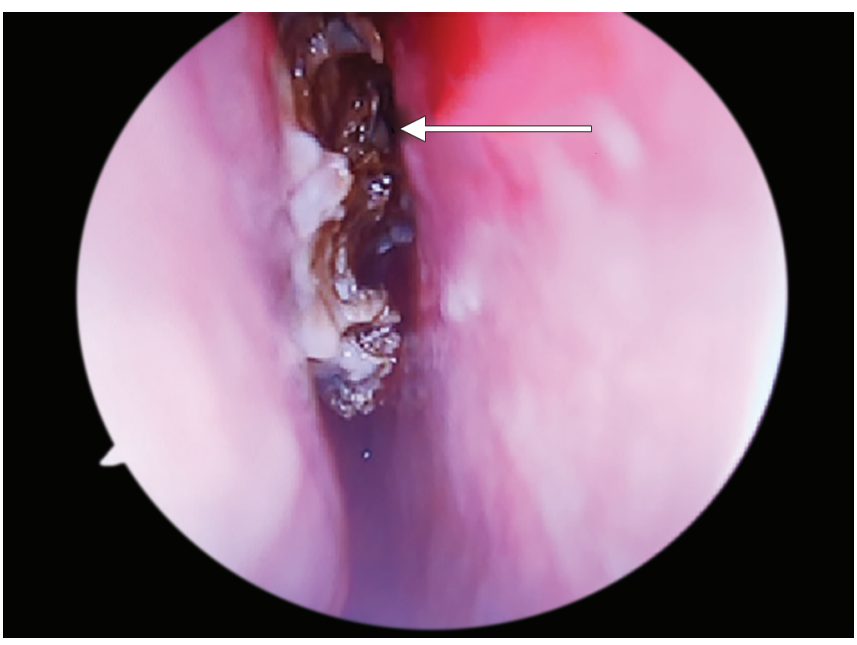

Fig. 2: Rhinolith in the left nasal cavity between the inferior turbinate and septum

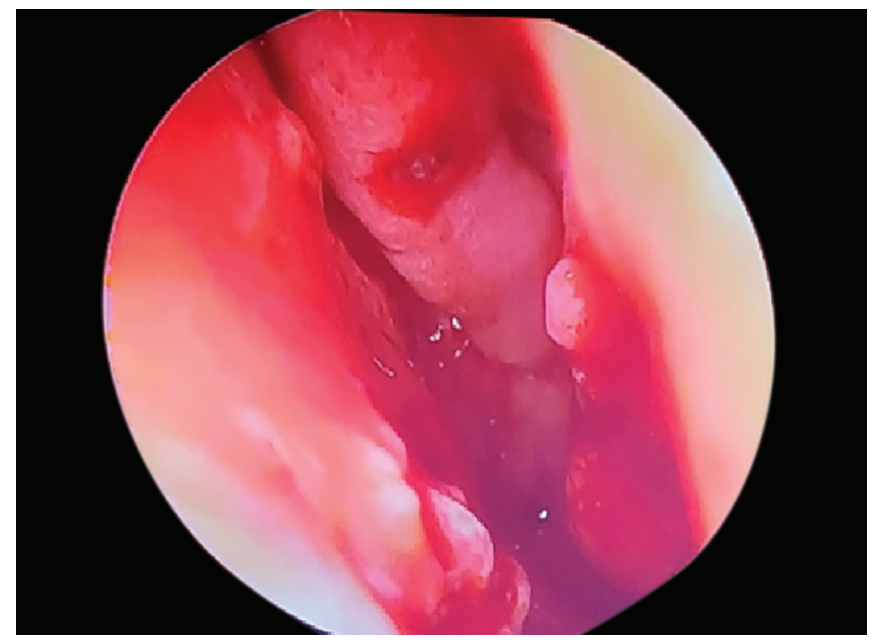

Fig. 4: Postoperative endoscopic examination revealing raw area over the inferior turbinate

on probing gave a gritty sensation. It was adherent to the septum and the inferior turbinate just below the anterior end of middle turbinate (Fig. 2). The nasal septum was found to be deviated on the right side. On the basis of the history, clinical examination, and imaging, a diagnosis of left-sided rhinolithiasis was established. The rhinolith was broken into pieces and taken out piecemeal avoiding much trauma to the mucosa (Fig. 3). Hemostasis was achieved with local adrenaline and hemocoagulase packs, and the patient was discharged the same evening on oral antibiotics and decongestant nasal drops. On subsequent follow-ups the, the patient had no recurrence or synechiae formations (Fig. 4).

\section{Discussion}

Rhinoliths are rare nasal masses mostly unilateral with a female preponderance. ${ }^{3}$ The most common location is along the floor and inferior meatus. ${ }^{4}$ The origin of rhinolith is always from a nidus, which can be an endogenous source such as ectopic tooth in maxillary sinus, blood clots, thick secretions or exogenous like a cotton fiber, seed, and animate objects like leech., ${ }^{5,6}$ The pathogenesis is described as an initial inflammatory reaction to

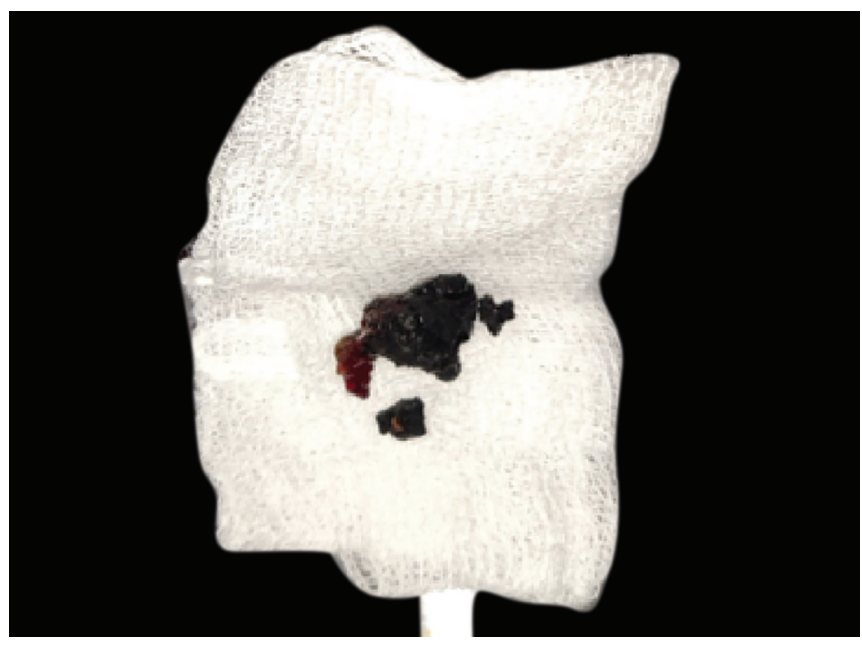

Fig. 3: Removed rhinolith

the nidus that in conjunction with obstruction and stagnation of the secretions leads to deposition of calcium carbonate and calcium phosphate, magnesium, iron, and aluminum, in addition to organic substances such as glutamic acid and glycine, resulting in a slow and progressive increase in size. ${ }^{7}$ Rhinoliths are gray to brown colored, foul-smelling, rough surfaced, and friable structures that are often situated in the anterior half of the nasal cavity on its floor. Most patients complain of purulent rhinorrhea and/or ipsilateral nasal obstruction. Other symptoms include fetor, epistaxis, sinusitis, headache, and, in rare cases, epiphora. ${ }^{8}$ In some patients, rhinoliths are discovered incidentally. In this case also, due to the lack of specific symptoms, the case was misdiagnosed and was reported late. This signifies that such uncommon entities must be kept in mind while examining such cases, and such a possibility should not be overlooked. The examination ought to embody anterior rhinoscopy and radiological evaluation. CT scan cannot differentiate a rhinolith from any other calcified mass, but it can detect related complications of rhinolith. CT scan of the paranasal sinuses can accurately determine the site and size of the rhinolith and identify any synchronal sinus disease that may require treatment. ${ }^{9}$ The treatment consists of the removal of the rhinolith, and the surgical approach that is chosen depends on the location and the size of the rhinolith and presence or not of complications; however, most of them may be removed endonasally. External approaches may be necessary in cases of giant rhinoliths, and endoscopes are extremely helpful in both approaches. ${ }^{9}$ Sometimes rhinoliths become so difficult to remove, that they may have to be broken into small pieces by forceps, ${ }^{10}$ or with the help of lithotripsy, ${ }^{11}$ or by pushing the rhinolith posteriorly into the nasopharynx and removing it through the oral cavity. ${ }^{12}$ Sometimes, septoplasty and/ or turbinoplasty need to be done. ${ }^{13}$ In this reported case, the rhinolith was removed by endoscopic guidance. This signifies that the larger the size of the rhinolith, its removal becomes troublesome, and in such a case, no blind method should be adopted to avoid the complications which follow the removal. The complications that have been described as secondary to rhinoliths include recurrent sinusitis, destruction of the nasal mucosa, oroantral and oronasal fistulas, septal deviation and perforation, palatal perforation, ${ }^{2}$ bony destruction of the maxillary sinus, frontal osteomyelitis, and 
epidural abscess. ${ }^{14}$ This signifies that the early the diagnosis, the better the outcome. The differential diagnosis of radiopaque nasal lesions includes a number of benign and malignant pathologies such as rhinolith, calcified polyp, ossifying fibroma, odontogenic tumor, osteoma, osteosarcoma, osteomyelitis, and carcinoma. ${ }^{12}$ Recurrence following the removal of the rhinolith has not been reported in the literature so far.

\section{Conclusion}

Although rhinoliths are rare, clinicians ought to remember the possibility of its incidence. It requires a high index of suspicion when dealing with nasal symptoms, such as progressive unilateral nasal obstruction, rhinorrhea (usually purulent and fetid), cacosmia, and unilateral nasal bleeding that favor the possibility of foreign bodies in children and rhinoliths in adults.

\section{Clinical Significance}

Presentation of rhinolith and diagnostic dilemma are discussed in this article so as to enable the attending clinician to be aware of this still prevailing condition and to prevent complications, such as oroantral and oronasal fistulas, septal perforation, palatal perforation, ${ }^{2}$ bony destruction of the maxillary sinus, frontal osteomyelitis and epidural abscess.

\section{References}

1. Appleton SS, Kimbrough RE, Engstrom HI. Rhinolithiasis: a review. Oral Surg Oral Med Pathol 1988;65(6):693-698. DOI: 10.1016/00304220(88)90012-6.
2. Ozcan I, Ozcan KM, Ensari S, et al. Rhinolithiasis with a nasal polyp: a case report. Ear Nose Throat J 2008;87(3):150-151. DOI: 10.1177/014556130808700310.

3. Kuczkowski J, Narozny W, Mikaszewski B, et al. Suppurative complications of frontal sinusitis in children. Clin Pediatr (Phila) 2005;44(8):675-682. DOI: 10.1177/000992280504400805.

4. Kharoubi S. Revue générale sur les rhinolithiases. Ann Otolaryngol Chir Cervicofac 2008;125(1):11-17. DOI: 10.1016/j.aorl.2007. 12.001.

5. Davis $\mathrm{O}$, Wolff A. Rhinolithiasis and maxillary antrolithiasis. Ear Nose Throat J 1985;64(9):421-426.

6. Kumar S, Dev A, Kochhar LK. Living leech in nose and nasopharynx: an unusual foreign body. Indian Journal of Otolaryngology 1989;41(4):160-161.

7. Seth S, Kumar H. Rhinolithiasis: A case report. J Clini Diagn Res 2011;5(4):856-858.

8. Orhan K, Kocyigit D, Kisnisci R, et al. Rhinolithiasis: an uncommon entity of the nasal cavity. Oral Surg Oral Med Oral Pathol Oral Radiol Endod 2006:101(2):28-32. DOI: 10.1016/j.tripleo.2005.07.036.

9. Hadi U, Ghossaini S, Zaytoun G. Rhinolithiasis: a forgotten entity. Otolaryngol Head Neck Surg 2002;126(1):48-51. DOI: 10.1067/ mhn.2002.121018.

10. Mustafa A, Nishori S. Rhinolith caused from the undetected foreign body: a case report. Kosova J Surg 2008;2(1):32-35.

11. Mink A, Gati I, Szekely J. Nasolith removal with ultrasound lithotripsy. HNO 1991;39(3):116-117.

12. Oguzhan D, Nurray BM, Osman KA. An unusual cause for nasal obstruction: rhinolithiasis. Bidder Tip Bilimleri Dergisi 2010;2(4):20-24.

13. Shah F, George S, Reghunanden N. A case presentation of a large rhinolith. Oman Med J 2010;25(3):230-231.

14. Atmacal S, Belet N, Sensoy G, et al. Rhinolithiasis: an unusual cause of sinusitis which is complicated with frontal osteomyelitis and epidural abscess. Turk J Pediatr 2010;52(2):187-190. 